\title{
Tuning metadata for better movie content-based recommendation systems
}

\author{
Márcio Soares \\ INESC TEC (formerly INESC Porto) \\ Campus da FEUP, Rua Dr. Roberto Frias, 378, 4200 - 465 Porto, Portugal \\ Tel.: +351222094000 \\ Fax.: +351222094050 \\ mmsoares@inescporto.pt \\ Paula Viana \\ INESC TEC (formerly INESC Porto) \\ Campus da FEUP, Rua Dr. Roberto Frias, 378, 4200 - 465 Porto, Portugal \\ Tel.: +351222094000 \\ Fax.: +351222094050 \\ ISEP/IPP - School of Engineering, Polytechnic Institute of Porto \\ Rua Dr. António Bernardino de Almeida, 431, 4200-072 Porto, Portugal \\ Tel.: +351228340500 \\ Fax.: +351228321 159 \\ paula.viana@inescporto.pt
}

\begin{abstract}
The increasing number of television channels, on-demand services and online content, is expected to contribute to a better quality of experience for a costumer of such a service. However, the lack of efficient methods for finding the right content, adapted to personal interests, may lead to a progressive loss of clients. In such a scenario, recommendation systems are seen as a tool that can fill this gap and contribute to the loyalty of users.

Multimedia content, namely films and television programmes are usually described using a set of metadata elements that include the title, a genre, the date of production, and the list of directors and actors. This paper provides a deep study on how the use of different metadata elements can contribute to increase the quality of the recommendations suggested. The analysis is conducted using Netflix and Movielens datasets and aspects such as the granularity of the descriptions, the accuracy metric used and the sparsity of the data are taken into account. Comparisons with collaborative approaches are also presented.
\end{abstract}

Keywords: recommendation algorithms; collaborative; content-based; metadata; 


\section{Introduction}

The proliferation of video distribution services contributed to the gradual increase of the number of available channels and on demand content. Overloaded with too much information, many viewers systematically give up watching a program and tend to jump between different channels or always watching the same one. Traditional tools such as the Electronic Program Guides (EPGs) still have strong limitations as usually only provide extensive lists of programs, which require the user to spend too much time searching for potentially interesting content. In this scenario, recommendation systems stand out as a possible solution to assist a watcher on the selection of the content that best fits his/her preferences.

In recent years, recommender systems (RS) have been used in quite different areas of application, including e-commerce (e.g. amazon), news and television services. In the context of multimedia services, Netflix ${ }^{1}, \mathrm{Hulu}^{2}$ and even $\mathrm{IMDB}^{3}$ have their own recommendation systems. These recommendation systems can take into account different aspects including information on the program itself, viewers' profiles and past shown interests or simply program popularity. Designing an accurate RS requires, as a first implementation decision, analysing the available data and deciding which parameters to be used given that they can affect the list of recommendations: can the recommendation of a film based on their actors be more effective than the recommended programs based on genre?; Programs described in more detail can provide more accurate recommendations?

Although work such as the one presented in $[16,18,41]$ has already discussed the thematic of performance and accuracy of recommendations algorithms, their main focus is on collaborative algorithms. Evaluation of content-based algorithms and how metadata elements may affect the results of TV RS has been little explored.

In this paper we compare the performance of collaborative and content-based algorithms using different metadata elements. The impact of using, independently, information on the genre, list of actors or directors or a more complete set of elements, on the quality and accuracy of the recommendation is analysed. Netflix and Movielens datasets are used to evaluate the performance of the different approaches in respect to accuracy metrics that include Mean Average Error (MAE) and Precision.

Given that there is no universal metadata scheme and that granularity of the different elements varies from application to application, it is also important to measure how these differences can influence the quality of the results. Based on this assumption, the paper

\footnotetext{
${ }^{1}$ www.netflix.com

${ }^{2}$ www.hulu.com

${ }^{3}$ www.imdb.com
} 
also provides results on how the use of different levels of sub-elements affects the quality of the RS.

Simulations have been run using different content-based algorithms and several partitions approaches of the main Netflix and Movielens datasets. The main results show that the conclusions of our work can be generalised and are not influenced by these issues.

\section{Related work}

Recommender systems are systems with the ability of providing suggestions or directing a person to a service, product or content, that has a potential of interest among a number of different alternatives [12,35]. Examples can be found in different domains including book $\left(\right.$ Amazon $\left.^{4}\right)$, music $\left(\right.$ Pandora $^{5}$, Last.fm ${ }^{6}$ ), video $\left(\right.$ Youtube $^{7}$ ) and product recommendation $\left(\mathrm{eBay}^{8}\right)$. Although the first recommendation systems date from the late 70 's, only in the early ' 90 s the first commercial applications of this type of systems were deployed [2].

In the multimedia domain, Netflix, a commercial service providing access to movies and TV shows, presents predicted ratings for every displayed movie in order to assist the user deciding on the service to rent. Movilens ${ }^{9}$ [32], a free, non-commercial, tool also provides services in the area of movie recommendations.

Television service providers have also demonstrated interest in enhancing their traditional programme guides and over the years several applications have emerged. The PTV project (Intelligent Personalized TV Guides) [15] was one of the first implementations in this area and is a reference to other solutions that came later.

A more sophisticated approach has been considered in [21], where not only historical information (e.g. ratings or gender preferences) is used but also information that can change in each access to the system (e.g. mood). The recommending mechanism is based on some user characteristics such as Activities, Interests, Moods, Experiences, and Demographic information (AIMED). Based on the idea that very often several people share the same living room and watch television at the same time, the work in [50] takes into account not a single user profile but handles a set of profiles in order to consider a group of people watching TV together.

\footnotetext{
${ }^{4}$ www.amazon.com

${ }^{5}$ www.pandora.com

${ }^{6}$ www.lastfm.com

${ }^{7}$ www.youtube.com

${ }^{8}$ www.ebay.com

${ }^{9}$ www.movielens.umn.edu
} 
Recommender systems are usually classified according to the approach that is used to find information that may fit the user's interest. The most popular recommendation approaches are [1]: (1) content-based filtering, (2) collaborative filtering and (3) hybrid.

Content-based systems try to recommend items that are similar to the ones that the user has demonstrate interest in the past. The similarity between the content is measured, in most cases, through the analysis of information that describes the contents, such as a film genre or the author of a book. The description of user's interests is obtained from information provided by the user himself, or alternatively by automatically creating his profile based on past actions.

Content-based systems can be built on text-based information about the items (keywords, title, genre, etc.) or on extracted features from the multimedia items. NewsWeeder [28], a content-based recommendation system for news on the Web, is an example of this type of RS: if the user demonstrates a preference for news related to sports, the system will recommend other news with sports content.

In collaborative algorithms, recommendations are based on the analysis of the similarity between users and performance is usually highly influenced by the number of active users in the system. GroupLens [36] was one of the first systems to adopt such algorithms. After reading netnews articles, users assign them numeric ratings which are later used to enable correlating users whose ratings are most similar and to predict how well users will like new articles, based on ratings from similar users.

Collaborative algorithms enjoyed a surge of interest with the Netflix Prize competition. Among the most popular approaches, the nearest neighbour methods on either users, items or both, and methods using matrix factorization (MF) [26,39] can be found. In fact, the Netflix Prize competition showed that advanced matrix factorization methods can be particularly helpful to improve the predictive accuracy of recommender systems. According to the recent progress in Collaborative Filtering (CF) techniques, current $\mathrm{CF}$ algorithms are primarily based on Neighbourhood Based Models (NBM) $[9,27]$ and MF with some variations [25,52,53], as the work presented in [51] where factors that can influence rating - such as mood, environment and time of day - are considered.

Content and Collaborative based algorithms are known to have advantages and disadvantages which Table 1 tries to summarize. By combining two or more recommendation techniques, hybrid approaches [12,29] try to improve system performance by reducing the disadvantages of each technique used individually. 
Table 1 Comparative analysis of Content and Collaborative RS [3].

\begin{tabular}{|c|c|c|}
\hline & Content-based algorithm & Collaborative filtering algorithm \\
\hline Advantages & $\begin{array}{l}\text { - Easy: it is easy to understand by user. } \\
\text { - Cheap: resource consumption for } \\
\text { computation is low. } \\
\text { - Light: non-personalized service is } \\
\text { possible without the user preference } \\
\text { profile. }\end{array}$ & $\begin{array}{l}\text { - Diversity: it can deal with any kind of } \\
\text { content. } \\
\text { - Serendipity: it provides items with } \\
\text { dissimilar content with those experienced } \\
\text { in the past. }\end{array}$ \\
\hline Disadvantages & $\begin{array}{l}\text { - Shallow: only a very shallow analysis of } \\
\text { certain kinds of content can be supplied. } \\
\text { - Over-specialization: the user is } \\
\text { restricted to seeing items similar to those } \\
\text { already experienced. }\end{array}$ & $\begin{array}{l}\text { - Sparsity: the lack of user preference data } \\
\text { causes a performance decline, and makes } \\
\text { it difficult to find nearest-neighbours for } \\
\text { users with peculiar taste. } \\
\text { - Scalability: increase of user preference } \\
\text { data leads to a performance improvement, } \\
\text { but much more resources are consumed. }\end{array}$ \\
\hline
\end{tabular}

The implementation of any of these approaches requires gathering information concerning the satisfaction of the users regarding the watched items. Two different approaches have been proposed: a classification range is defined and users are required to explicitly input their degree of enjoyment, or the system implicitly infers user's preferences by monitoring his activity while using the service. In our previous work [43] we developed a web based recommender system that helps the user navigating on broadcasted and online television content. In this system the user profile is constructed using information collected both explicitly and implicitly. Explicit information corresponds to classifications given to watched programs (1 to 5). The user can however decide voluntarily not to assign any classification to a program and, in such a case, the system automatically ascertains the amount of time that he remains watching a program. This time is converted into a quantitative classification ranging from 1 to 5 and assumed as the rating that the user would have given to that program.

Recent work tries also to consider additional information in order to improve RS results. Contextual information [19,22,23,37,40] and social relations [14,24,45,48,49], that can be obtained e.g. from sensors in mobile devices and from social networks, have been used as further inputs to RS algorithms. This has been applied for different purposes, including tag recommendation systems used to improve metadata describing resources in the Internet $[14,30,47]$.

Although a lot of effort has been put on developing new algorithms and using additional information for RS, most of the work has been concentrated on CF and the way metadata elements affect the performance in $\mathrm{CB}$ approaches has been rarely explored. Some relevant work on this topic can be found in [31], [34], [38] and [44]. Lommatzsch [31] compares different approaches for aggregating semantic movie knowledge and 
discusses the gain of combining different metadata attributes. In [38], the authors investigate the value of movie metadata compared to movie ratings in regards to predictive power. They show that by using collected movie metadata, prediction performance for the implemented methods is comparable to $\mathrm{CF}$ and can be used to predict preferences on new movies. The integration of semantic and emotion information along with the ratings is analysed in [34]. Performance of these CF models is tested using different combinations of the features spaces, including movie metadata, for different training datasets constructed from the original Movielens data. Symeonidis [44] developed a feature-weighted user profile to disclose the duality between users and features. The main objective is to exploit the correlation between users and semantic features that should reveal the real reason of users' rating behaviour. The developed approach is compared against well-known $\mathrm{CF}$ and $\mathrm{CB}$, considering different metadata, and a hybrid algorithm with a real dataset.

The work presented in this paper adds new considerations in the area of $\mathrm{CB}$ approaches for movie recommendation and complements the previously published work. For validation purpose, we conducted simulations using two distinct datasets, namely, Movielens and Netflix. This allows result's generalizability, by confirming the achievements in independent samples, which was not provided in previously related work. Furthermore, given that metadata attributes can contain different levels of granularity (e.g. for the Netflix dataset, the movie genre is described in much more detail), and that results could be affected by this, rather than by the metadata element by itself, we also conduct different experiments that enable eliminating this hypothesis. The impact of the datasets sparsity is also deeply evaluated and, as a result, it's quite likely that the results presented in our study can be generalized to all datasets and metadata schemas within the field of movies and multimedia programs.

Finally, we also examine how many of top-N recommended items are the same for each of the studied cases. For example, suppose an algorithm that uses directors and another that uses genre to make recommendations and have the same performance. Will they both recommend the same items at top-10?

\section{Recommendation approaches}

Two recommenders, that implement collaborative-based and content-based approaches, were implemented based on the work described in [1,42]. This enables comparing performances and to investigate if content-based systems can approach or even outperform the collaborative algorithm. Given that the aim of this work is to thoroughly analyse the use of metadata in CB algorithms, rather than comparing the performance of $\mathrm{CF}$ approaches, tests were run using a standard implementation based on the nearest 
neighbour for the $\mathrm{CF}$ algorithm and two implementations of $\mathrm{CB}$ systems, namely a nearest neighbour and a genre learning process. The next sections briefly describe each of the algorithms.

\subsection{Nearest neighbour collaborative algorithm}

The main objective of the user-to-user collaborative filtering technique is to estimate the rating that a user $u$ would assign to a particular item $i$ based on ratings assigned to that same item by other users having a profile similar to the user $u$ under consideration. Being $R\left(u^{\prime}, i\right)$ the rating that user $u^{\prime}$ (similar to user $u$ )gave to item $i$, the rating to be calculated, represented by $R(u, i)$, is given by:

$$
\mathrm{R}(\mathrm{u}, \mathrm{i})=\frac{\sum_{\mathrm{u}^{\prime} \in \mathrm{N}(\mathrm{u})} \operatorname{sim}\left(\mathrm{u}, \mathrm{u}^{\prime}\right) \cdot \mathrm{R}\left(\mathrm{u}^{\prime}, \mathrm{i}\right)}{\sum_{\mathrm{u}^{\prime} \in \mathrm{N}(\mathrm{u})}\left|\operatorname{sim}\left(\mathrm{u}, \mathrm{u}^{\prime}\right)\right|}
$$

$N(u)$, the set of users considered similar to user $u$ (user neighbours), can range from one to all users in the dataset. Limiting the size to some specific number (e.g. two) will determine how many similar users will be used in the computation of the rating prediction $R(u, i)$.

The similarity between two users, $\operatorname{sim}\left(u, u^{\prime}\right)$, can be calculated using different metrics. In our implementation, the cosine similarity was used:

$$
\operatorname{sim}\left(\mathrm{u}, \mathrm{u}^{\prime}\right)=\frac{\sum_{i=1}^{n} \mathrm{R}(\mathrm{u}, \mathrm{i}) \mathrm{R}\left(\mathrm{u}^{\prime}, \mathrm{i}\right)}{\sqrt{\sum_{i=1}^{n} \mathrm{R}(\mathrm{u}, \mathrm{i})^{2}} \sqrt{\sum_{i=1}^{n} \mathrm{R}\left(\mathrm{u}^{\prime}, \mathrm{i}\right)^{2}}}
$$

\subsection{Content-based algorithms}

In order to try generalising the results for different CB approaches, simulations using two different algorithms presented in the literature were conducted. The next sections briefly describe each of the methods.

\subsubsection{Nearest neighbour content-based}

Content-based approaches estimate the similarity between items, using metadata information that describes them. In our work, different distance measures were used in the simulations, depending on the metadata element under consideration.

When comparing words' sequences where the order is not relevant, the cosine distance was used. One example is the analysis of the genre of a movie, where \{Romance,Comedy is considered alike to \{Comedy,Romance\}.

For other metadata attributes, as the list of actors or directors, in which the order may have some relevance, the Inverse Rank Measure was used. This metric calculates the similarity between two sequences taking into account the order of the elements and 
assigning different weights depending on the position of each element, according to the following expressions [4]:

$$
\begin{gathered}
\mathrm{N}^{\left(\mathrm{k}_{1}, \mathrm{k}_{2}\right)}\left(\sigma_{1}, \sigma_{2}\right)=\sum_{\mathrm{Z}}\left|\frac{1}{\sigma_{1}(i)}-\frac{1}{\sigma_{2}(i)}\right| \\
+\sum_{\mathrm{S}}\left|\frac{1}{\sigma_{1}(\mathrm{j})}-\frac{1}{k_{2}+1}\right| \\
+\sum_{\mathrm{T}}\left|\frac{1}{\sigma_{2}(\mathrm{j})}-\frac{1}{k_{1}+1}\right|
\end{gathered}
$$

where $Z$ is the set of the overlapping elements, $\sigma_{1}(i)$ is the rank of document $i$ in the first set and $\sigma_{2}(i)$ is its rank in the second set (both ranks are defined for elements belonging to $Z$ ). In addition, $S$ is the set of documents that appear in the first list but not in the second, while $T$ is the set of elements that appear in the second list, but not in the first [5]; $k_{1}$ and $k_{2}$ are the number of elements of each set.

This measure is normalized as follows:

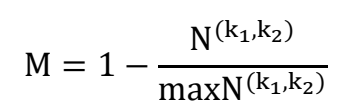

where

$$
\operatorname{maxN}^{\left(\mathrm{k}_{1}, \mathrm{k}_{2}\right)}=\sum_{\mathrm{i}=1}^{\mathrm{k}_{1}}\left|\frac{1}{\mathrm{i}}-\frac{1}{\mathrm{k}_{2}+1}\right|+\sum_{\mathrm{i}=1}^{\mathrm{k}_{2}}\left|\frac{1}{\mathrm{i}}-\frac{1}{\mathrm{k}_{1}+1}\right|
$$

For the example in Table 2, this metric results in a value of 0.31 for the pair Movie 1/Movie 2 and of 0.62 for Movie 1/Movie 3 as illustrated below for the first case:

$\delta_{1}=(A, B, C, D)$

$\delta_{2}=(E, A, B)$

$\mathrm{N}^{\left(\mathrm{k}_{1}, \mathrm{k}_{2}\right)}\left(\sigma_{1}, \sigma_{2}\right)=\left(\left|\frac{1}{1}-\frac{1}{2}\right|+\left|\frac{1}{2}-\frac{1}{3}\right|\right)+\left|\frac{1}{3}-\frac{1}{3+1}\right|+\left|\frac{1}{1}-\frac{1}{4+1}\right| \approx 1.54$

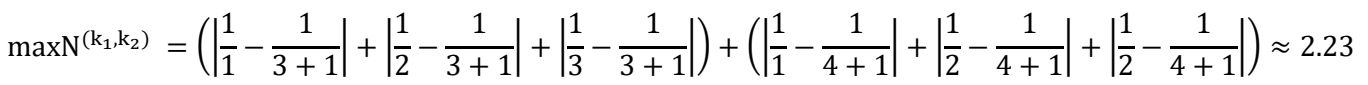

$\mathrm{M} \approx 0.31$

Although Movie 2 has two mutual actors with Movie 1 (A and B), while Movie 3 only shares actor A with Movie 1, this is in a more prominent position. Thus, Movie 3 is considered, by the Inverse Rank Measure, to be more similar to Movie 1 since they have the same main actor.

Table 2 Example of movies and actors to exemplify Inverse Rank Measure

\begin{tabular}{cc}
\hline Movie & Actors \\
\hline Movie 1 & A, B, C, D \\
Movie 2 & E, A, B \\
Movie 3 & A, F, G, H \\
\hline
\end{tabular}


The final similarity between the items under analysis is obtained by weighting, with different factors $\left(p_{a}\right)$, the individual values obtained for each of the attributes considered (such as genre, actors and directors) as presented in (6).

$$
\operatorname{sim}\left(\mathrm{i}, \mathrm{i}^{\prime}\right)=\frac{\sum_{\mathrm{a} \in \mathrm{A}} \operatorname{sim}_{\mathrm{a}}\left(\mathrm{i}, \mathrm{i}^{\prime}\right) * \mathrm{p}_{\mathrm{a}}}{\sum_{\mathrm{a} \in \mathrm{A}} \mathrm{p}_{\mathrm{a}}}
$$

Being $i^{\prime}$ an item similar to item $i$ (not yet rated), $\operatorname{sim}\left(i, i^{\prime}\right)$ the similarity between items, and $R\left(u, i^{\prime}\right)$ the rating that the user $u$ assigned to $i^{\prime}$, the rating that user $u$ will give to item $i$, is given by:

$$
\mathrm{R}(\mathrm{u}, \mathrm{i})=\frac{\sum_{\mathrm{i}^{\prime} \in \mathrm{N}(\mathrm{i})} \operatorname{sim}\left(\mathrm{i}, \mathrm{i}^{\prime}\right) \cdot \mathrm{R}\left(\mathrm{u}, \mathrm{i}^{\prime}\right)}{\sum_{\mathrm{i}^{\prime} \in \mathrm{N}(\mathrm{i})}\left|\operatorname{sim}\left(\mathrm{i}, \mathrm{i}^{\prime}\right)\right|}
$$

\subsubsection{Genre Learning Technique}

Items are often grouped into one or more categories such as genres or actors of movies and TV programs, or authors of books. In attribute-based prediction techniques, each attribute has an importance weight that can vary per user. Based on this importance weights' predictions can be generated. GenreLMS [42] learns how interested a user is in the genre, actors, directors or other attributes assigned to items and calculates a prediction using a linear function over different attributes (Equation (8)).

$$
\mathrm{P}=w_{0}+\sum_{a=0}^{n} w_{a} x_{a}
$$

For each attribute $a$ the algorithm learns a weight $w_{a}$ indicating the relative importance of each attribute to the user, whereas $w_{0}$ is a constant value for the user. The extent (percentage) to which attribute $a$ belongs to the item, is indicated by $x_{a}$, with:

$$
\sum_{a}^{n} x_{a}=1
$$

Learning weights for each attribute takes place the moment a user rates an item. The learning algorithm uses the basic least Mean Square Method [33] (originating the name LMS used for this technique). With LMS, each weight is updated using the difference between the actual rate $R$, provided by the user, and the predicted rate $P$ :

$$
w_{a_{n e w}}=w_{a}+\mu(R-P) x_{a}
$$

here $\mu$ is a constant moderator determining the rate in which weights are updated. 


\section{Metrics for performance evaluation}

Several metrics have been proposed to evaluate the performance of recommenders $[8,17,20,46]$. One of the most commonly used approaches is the Mean Absolute Error (MAE) that calculates the difference between the classification predicted by the system and the real rating assigned by the user to this same item, providing and estimation of the average error associated to recommendations (Equation (10)).

$$
\text { MAE }=\frac{1}{n} \sum_{i=1}^{n}\left|p_{i}-a_{i}\right|
$$

Although MAE is widely used due to its simplicity, it may not be appropriate for evaluating the quality of the top $\mathrm{N}$ recommendations $[11,20]$ as performance analysis should focus on the list of recommendations provided to the users (the top $\mathrm{N}$ items with the highest potential).

When not interested in the exact prediction value, but only in finding out if the active user will like or not the current item, classification accuracy metrics can be used. Widely used in binary classification systems, this metrics try to estimate whether the like/dislike estimated classification, matches the real user tastes rather than to analyse the exact value of the prediction. This approach may also be used in n-nary classification systems, by using an appropriate threshold that converts the results to a two level system. For instance, for a rating scale in the range 0 to 5 , classifications above 4 could be considered as a like and below as a dislike.

To evaluate how well a recommendation list match the user's preferences, precision is commonly used $[15,20]$. Items are first classified according to their real importance to the user and their place in the list of results provided by the system:

- True Positive (TP) - an interesting item is recommended to the user;

- True Negative (TN) - an uninteresting item is not recommended to the user;

- False Negative $(F N)$ - an interesting item is not recommended to the user;

- False Positive (FP) - an uninteresting item is recommended to the user.

Two classes of recommendations - good and bad [20] - are then defined as illustrated in Table 3 .

Table 3 - Classification of the recommendation results

\begin{tabular}{c|c|c} 
& Recommended & Not recommended \\
\hline Actually good & TP & FN \\
\hline Actually bad & FP & TN
\end{tabular}


The Precision of a set of recommendations indicates the correct classification percentage and is given by:

$$
\text { Precision }=\frac{\mathrm{TP}}{\mathrm{TP}+\mathrm{FP}}
$$

Examples of application of precision are presented in $[6,10]$ or [41].

\section{Datasets}

\subsection{Datasets characterization and enhancement}

Two datasets were used in the evaluation of the results presented in this paper: the Movielens 10M and the Netflix Prize datasets.

Movielens $10 \mathrm{M}^{10}$ uses a rating scale in the range [1..5] and contains $10,000,054$ ratings to 10,681 movies by 71,567 users. Each user rated at least 20 items and the average number of rating per user is 143 .

Netflix dataset contains program ratings assigned by the costumers of the ondemand Internet streaming service. This dataset is composed of 100,480,507 ratings that 480,189 users gave to 17,770 movies. The rating scale adopted is also in the range of $[1 \ldots 5]$ and all the users rated at least 1 movie. The average number of ratings per user is 35.

These datasets have been previously used for comparing the performance of collaborative based recommendation systems. However, given that the purpose of the work presented in this papers is to analyse the impact of metadata in the construction of recommendation algorithms, additional information had to be added since these datasets only contain the classifications given by users to the programs, and do not have program description attributes (genres, actors and directors).

Figure 1 illustrates the process used for enhancing these two datasets. Metadata elements available from the Netflix ${ }^{11}$ and IMDB $^{12}$ APIs were extracted and used to complement the initial available data. Given that two different data sources were used, some discrepancies can be noticed, for example, in the list and number of genres used to describe a film.

\footnotetext{
${ }^{10} \mathrm{http}: / /$ movielens.umn.edu/

${ }^{11} \mathrm{http}: / /$ developer.netflix.com/

12 http://www.imdbapi.com/
} 


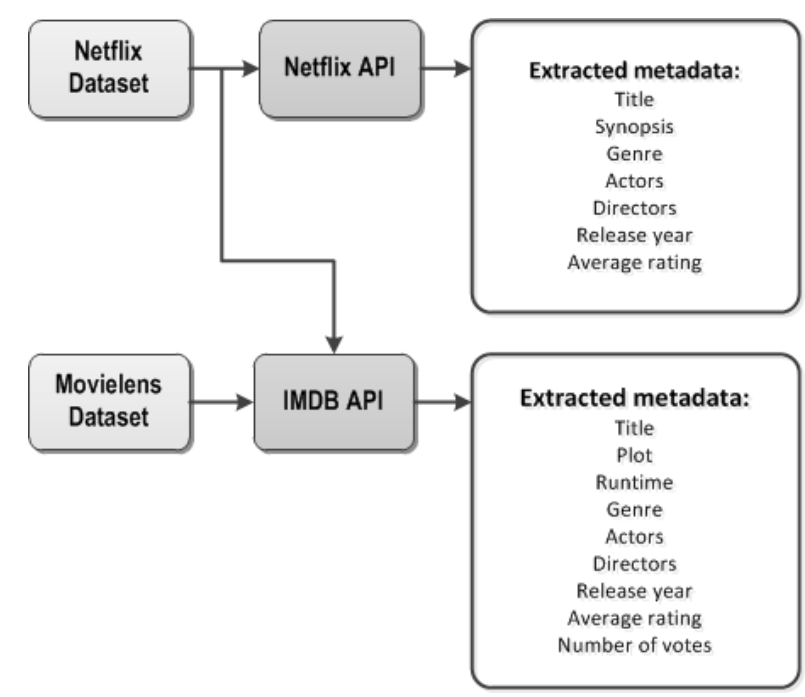

Figure 1 - Enhancement of Netflix and Movielens datasets with metadata

Table 4 presents the number of available genres, together with some examples that illustrate the differences between each of the datasets considered. Besides the considerable difference in the number of available genres (26 genres for describing programs in IMDB compared with 270 for Netflix), programs from Netflix are described with much more detail. For the class of horror programs, for example, IMDB provides only one possibility while Netflix allows horror films to be subcategorized using 3 genres.

Table 4 - Example of available genre classifier in IMDB and Netflix

\begin{tabular}{ccc}
\hline & & Dataset \\
& IMDB & Netflix \\
\hline \multirow{2}{*}{ Number of types of genres } & 26 & 270 \\
\hline \multirow{2}{*}{ Genre } & Drama & Dramas \\
& Sci-Fi & Romantic dramas \\
& Action & Dramas based on a book \\
& Comedy & Sci-Fi \& Fantasy \\
& Adventure & Fantasy \\
& Horror & Horror movies \\
Crime & Supernatural horror movies \\
& Family & Italian horror movies \\
\hline
\end{tabular}

Given these differences, a third dataset based on Netflix original ratings but enhanced with IMDB metadata was built. This enables conducting tests and comparing results that better illustrate the influence of the different metadata standards in the results of recommendation algorithms. 


\subsection{Datasets partitioning approaches}

Since the datasets contain a large set of ratings (a few millions) a dataset resizing was done to reduce computational costs. This process took into account some factors directly related to the aspects under consideration in this work. Given that content-based filtering will be an important component in this study, for each dataset, only programs whose actors and directors are present in at least two programs were selected. For example, if the actor Harrison Ford, the central actor of the movie Indiana Jones does not appear in at least two more films, Indiana Jones movie is eliminated.

After this initial filtering, and still with the objective of reducing the size of the dataset, two alternative approaches were used: (i) based on the number of ratings given by users to programs; (ii) according to a pre-defined test time used as the border between historical information and future ratings to be predicted.

For approach (i), the top 3000 users, that is, the ones that contributed with more ratings, were selected. These 3000 users were further split into three groups (first three rows in Table 5), according to the percentage of ratings. To further study the impact of sparsity in the results, a highly sparse dataset was constructed considering the 3000 users with less ratings in each of the dataset. Table 5 summarises the main characteristics of the sub-datasets used.

Table 5 - Characterisation of the Sub-datasets constructed for the experiments (based on the number of ratings)

\begin{tabular}{ccc|ccc}
\hline \multicolumn{5}{c}{ Dataset } \\
\hline Name & Movielens & Sparsity & Name & Netflix & Sparsity \\
\hline ml_25(1) & $\begin{array}{c}1114 \text { users, } \sim 25 \% \text { of } \\
\text { all the ratings }\end{array}$ & $94 \%$ & nflx_25(1) & $\begin{array}{c}972 \text { users, } \sim 25 \% \text { of all } \\
\text { the ratings }\end{array}$ & $89 \%$ \\
\hline ml_25(2) & $\begin{array}{c}872 \text { users, } \sim 25 \% \text { of all } \\
\text { the ratings }\end{array}$ & $93 \%$ & nflx_25(2) & $\begin{array}{c}874 \text { users, } ~ 25 \% \text { of all } \\
\text { the ratings }\end{array}$ & $88 \%$ \\
ml_50 & $\begin{array}{c}1025 \text { users, } ~ \\
\text { all the ratings }\end{array}$ & $87 \%$ & nflx_50 & $\begin{array}{c}1197 \text { users, } ~ \\
\text { all the ratings }\end{array}$ & $80 \%$ \\
\hline ml_s & 3000 users & $99 \%$ & nflx_s & 3000 users & $99 \%$ \\
\hline
\end{tabular}

This approach for partition a dataset to allow reducing the costs of experimentation have been used in other published work [40]. However, it does not accurately represent the actual behaviour of a real world recommendation system: at a given time, recommendations should only use historical/known information from the past. Taking this aspect into account, an even cheaper alternative to split large datasets is to define a date to be considered as the border between past and future [40]: if a particular user rated 
a program on January 1, recommendation algorithms can only compare this program with programs that user rated before the day specified.

For our tests, the borderline was defined as the $1^{\text {st }}$ of January 2005 and, as result, the original dataset was divided in two parts: (a) a list of programs for which available ratings date for after 2005 and for which we will try to make recommendations; and (b) programmes that were rated before 2005 and from which we will considerer the attributes (ratings and metadata) to make recommendations. The characterization of each of the datasets obtained is presented in Table 6 .

Table 6 - Characterisation of the Sub-datasets constructed for the experiments (chronological division)

\begin{tabular}{lcc}
\hline & Movielens & Dataset \\
\hline Date rating range & $1995 \ldots 2009$ & Netflix \\
\hline Time test & 2005 & $1999 \ldots 2005$ \\
\hline Number of Users & 20,278 & 2005 \\
\hline Number of Items & 10,580 & 18,899 \\
\hline Number of Ratings & $3,430,566$ & 2,783 \\
\hline
\end{tabular}

\section{Simulations and results}

The main objective of the present work was to analyse the influence of some parameters in the recommendation process. For that, a set of simulations was conducted in order to enable:

- Comparing collaborative and content-based algorithms' performance;

- Checking how different metadata elements, used for computing the similarity between items in the content-based approach, influence the quality of obtained recommendations. Simulations using individually the genre, the list of actors or the list of directors were done. A final simulation considered the use of all the three elements together as well as all the possible two by two combinations;

- Analysing the use of different metrics on the evaluation of the performance of the algorithms (MAE and Precision);

- Checking how different metadata schemas having different granularity (in the case of this work, genre), influence the quality of obtained recommendations;

- The analysis of the sameness of the top-N predictions for the collaborative and content-based algorithms.

The tests were conducted for both the modified Netflix and Movielens datasets as described in Section 5. Figure 2 summarizes the metadata schema used in the simulations. 


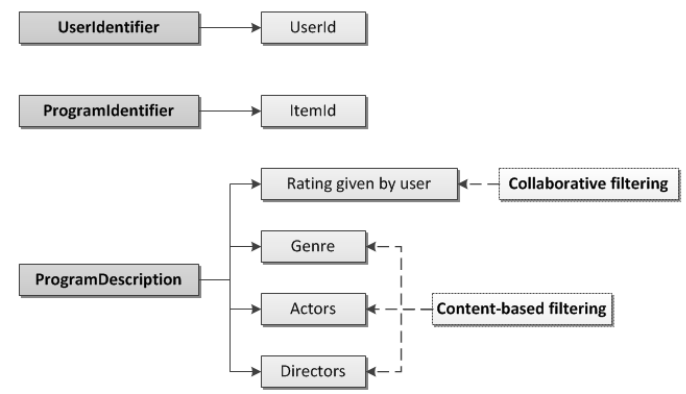

Figure 2 Metadata schema

\subsection{Impact of metadata attributes in algorithms' performance}

To evaluate the performance of the collaborative and content-based algorithms two metrics were used: MAE and Precision. MAE measures how close the predicted results are to the user's real ratings and considers all the predictions made by the system for each user, while precision measures the ability of the algorithms to only recommend what is relevant. Since metrics as Precision are optimised to evaluate the top-N recommendation list, the top 10 recommendations were considered for calculating this evaluation parameter.

Figure 3 and Figure 4 depict the MAE and Precision results on the Netflix and Movielens sub-dataset partitioned according to approach (i) described in section 5.2.

\section{MAE - Netflix}

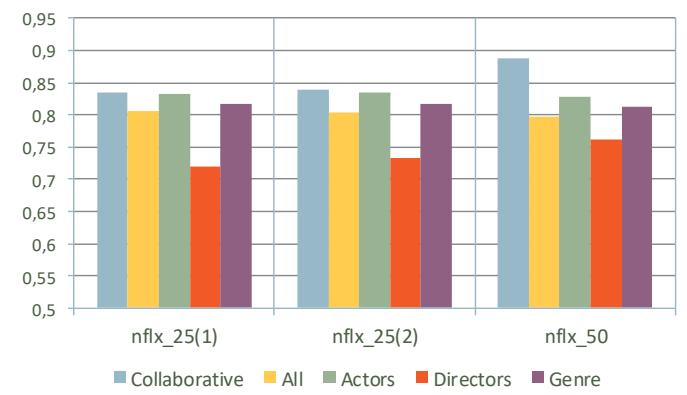

\section{Precision - Netflix}

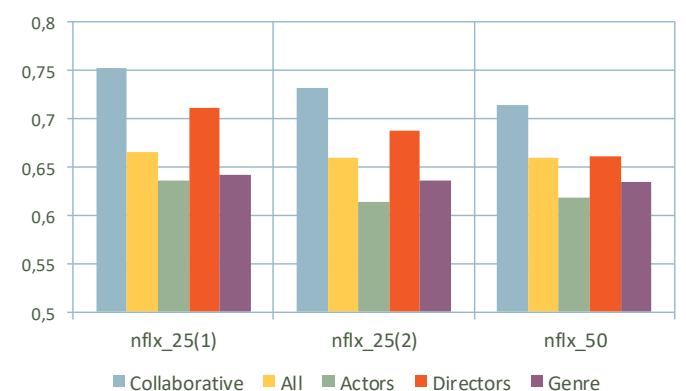

Figure 3 - MAE and Precision for the Netflix dataset (partition based on the number of ratings)

MAE - Movielens

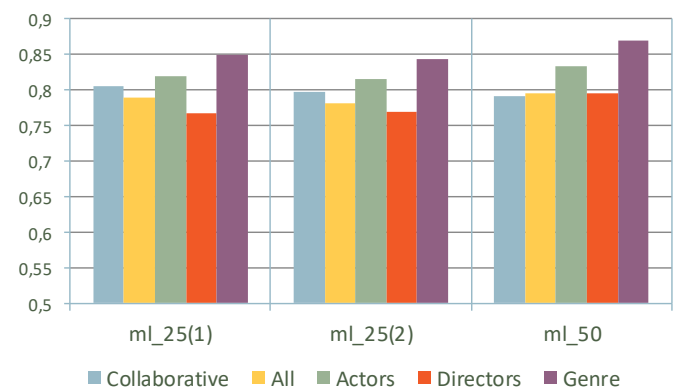

\section{Precision - Movielens}

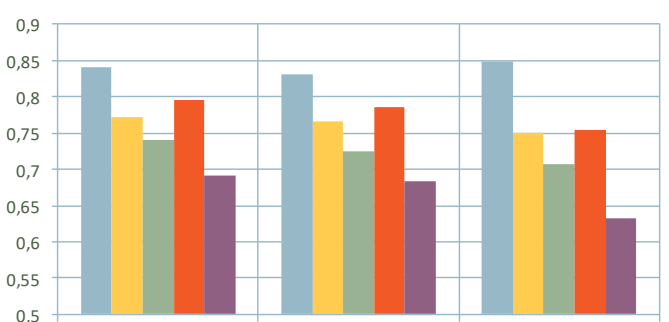

$\mathrm{ml} \_25(1)$

$\mathrm{ml} \_25(2)$

$\mathrm{ml} \_50$

Figure 4 - MAE and Precision for the Movielens dataset (partition based on the number of ratings) 
One of the conclusions that can be drawn from the results presented is that using the information on directors rather than the, commonly considered relevant, genre and list of actors, enables a better performance of content-based algorithms. Moreover, the impact on using a more complete set of metadata (All: Actors+Directors+Genre) does not contribute to decrease MAE and may only slightly contribute to increase the precision. This observation is relevant since by using only one metadata element (the directors) the algorithm becomes less computationally expensive.

When comparing the two approaches for evaluating the performance of the algorithms, it is important to notice that the metric used can have some influence when comparing content-based and collaborative filtering: while the collaborative algorithm achieves a better performance for the Top-10 precision, the prediction error based on MAE is smaller for the content-director-based approach.

One of the aspects that this study proposed to analyse was how the sparsity of the dataset (small number of ratings or large number of new items) would affect the results. Results in Figure 3 and Figure 4 show similar behaviour independently of the dataset partition considered. However, given that these sub-datasets were constructed based on the users with more rated movies, which makes sub-datasets quite similar, tests using a sub-dataset with high sparsity (ml_s and nflx_s datasets in Table 5) were performed. As expected and illustrated in Figure 5, some decrease of the collaborative algorithm's performance for both tested datasets is noticed. The most relevant conclusion is, however, that $\mathrm{CB}$ approach based on the directors' information significantly outperforms the $\mathrm{CF}$ algorithm and is not negatively influenced by the sparsity. This conclusion provides important guidelines to deal with the cold start problem and to enable new items to be recommended.
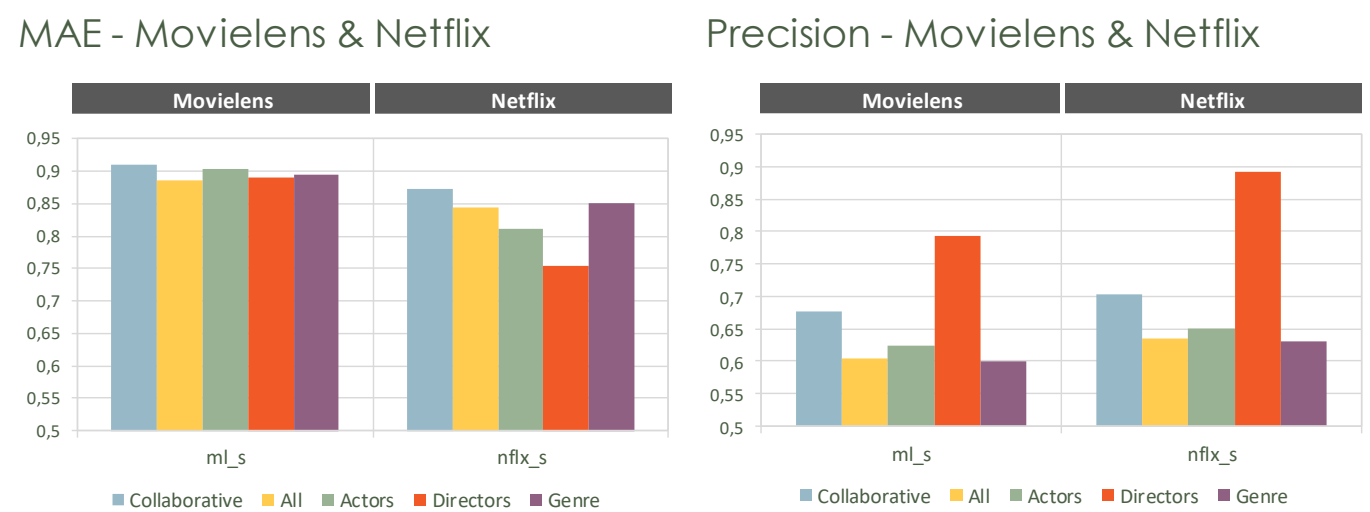

Figure 5 - MAE and Precision for the Movielens and Netflix sparse sub-dataset

Considering the Netflix and Movielens sub-dataset partitioned according to approach (ii), the results obtained were close to the ones achieved before as shown in Figure 6 . This confirms that the main conclusions are not influenced by the way the test 
dataset is constructed (either using the entire user profile history or just knowing his past preferences).

\section{MAE}

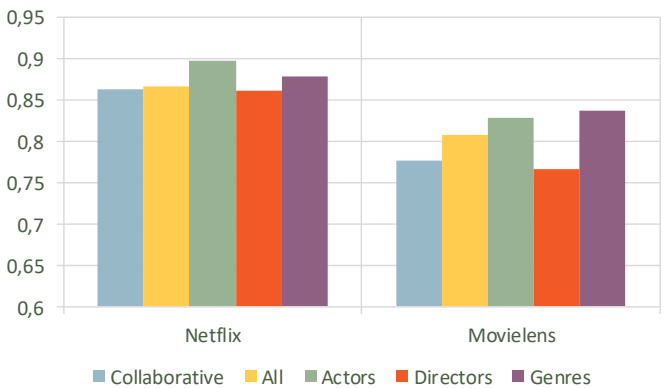

\section{Precision}

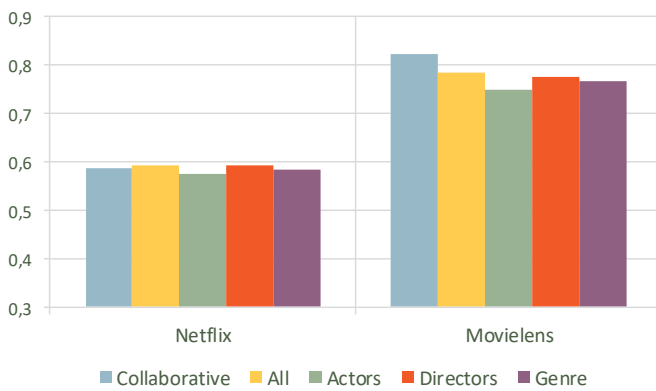

Figure 6 - MAE and Precision for Netflix and Movielens datasets (chronological partition).

Figure 7 provides additional results that enable evaluating the impact of all the possible combinations of two metadata elements. The results show that, by combining metadata information, performance can be improved for the less relevant attributes (e.g. genre). However, directors individually still outperform all the combinations. This conclusion is rather important due to the fact that using additional information results in more computational costs that are not converted into performance gain.

\section{MAE \& Precision - Movielens \& Netflix}
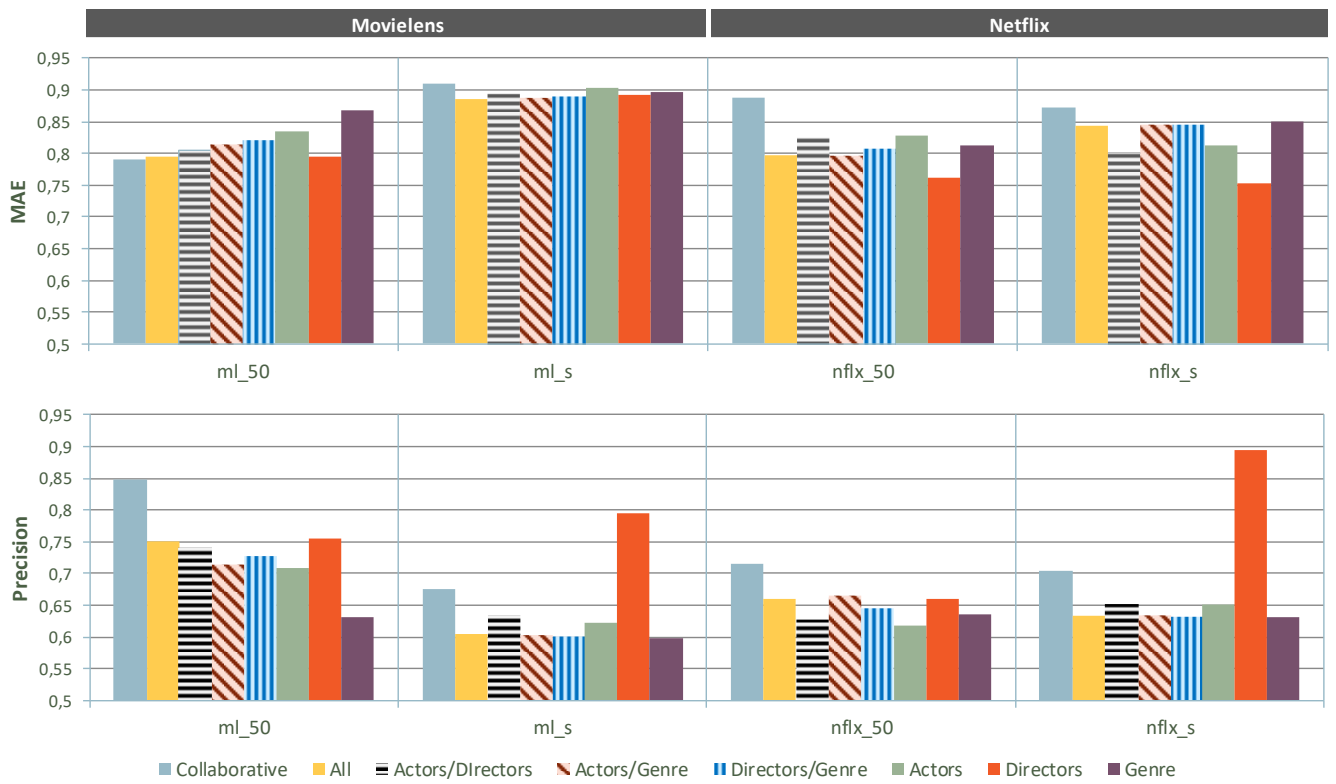

Figure 7 - Comparative study of all the possible combinations among metadata elements. 
In order to further study the influence of the different metadata elements in contentbased RS, additional tests using a different algorithm (GenreLMS) were made. For the results depicted in (Figure 8) we considered an optimal update moderator value $(\mu)$ calculated for each of the simulations.

\section{MAE \& Precision - Movielens \& Netflix}

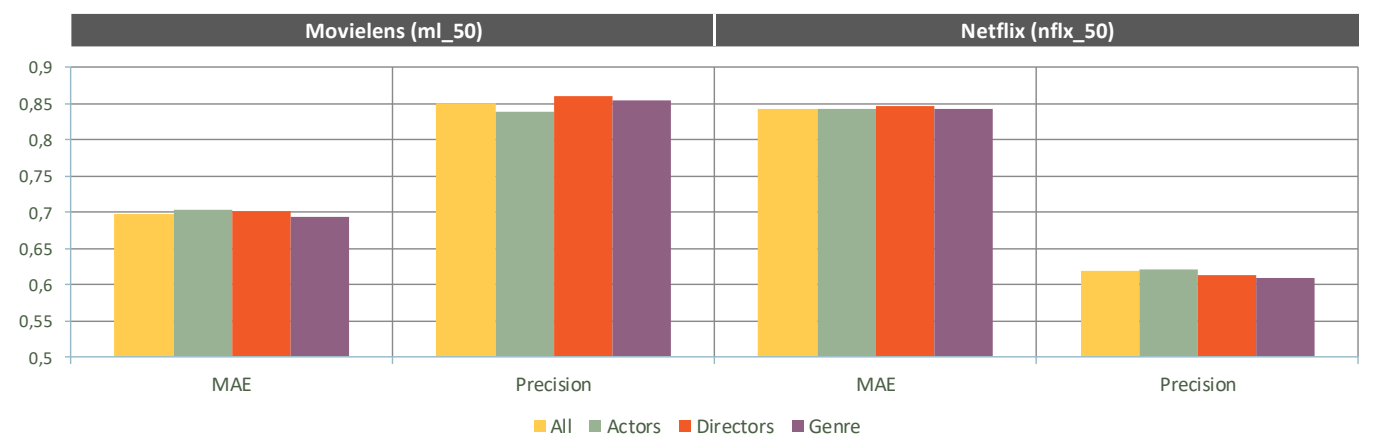

Figure 8 - MAE and Precision for Movielens and Netflix sub-datasets for the GenreLMS algorithm.

The immediate conclusion that could be drawn from the pictures is that the GenreLMS algorithm is not noticeably affected by the metadata used. This might be regarded as a different behaviour when compared to the previous results. However, given that the execution time of this algorithm is highly affected by the number of attributes used, these results should be carefully analysed. Considering the Netflix results, one can conclude that by using all the metadata attributes, the results are slightly improved. However, given that this improvement is achieved at the expense of a great execution time and that the difference in performance towards using the directors' information is almost unnoticeable, the best attribute to be used can still be considered the director. As a similar analysis can be done for the Movielens dataset, the final conclusion is still that the directors attribute provide the best information to be used in CB algorithms.

\subsection{Impact of the genre granularity on algorithms' performance}

Although quite a lot of effort has been put on the standardization of a multimedia description schema, different solutions coming from different organizations and having different levels of details were published. Not only public metadata schemas like TVAnytime, MPEG-7 or SMPTE are available, but private solutions customized to fulfil individual requirements such as the ones used by Netflix and IMDB are also used.

The list of available genres or the use of a main genre and a set of sub-genres illustrates how differently a programme can be described. Table 7 exemplifies how the same content is described in IMDB and Netflix. 
Table 7 List of genres used by Netflix and IMDB for the film "Alien vs Predator"

\begin{tabular}{ccc}
\hline Movie & IMDB genre & Netflix genre \\
\hline & Action & Action \& Adventure \\
& Adventure & Horror Movies \\
Alien vs. Predator & Sci-Fi & Sci-Fi \& Fantasy \\
& & Monster Movies \\
& & Action Sci-Fi \& Fantasy \\
& Alien Sci-Fi \\
& Sci-Fi Horror \\
\hline
\end{tabular}

In order to analyse how this difference would affect the results, a new dataset was assembled: for the Netflix dataset, genre has been replaced by the genre of the IMDB database. New tests were performed for the nearest neighbour content-based algorithm using all metadata attributes and using the gender only.

Figure 9 and Figure 10 compare the results obtained for the original and modified Netflix datasets. It is clear that the increase in the number of genres used to describe content enables better results, showing how the granularity of the metadata schema can influence the quality of the recommendation in content-based approaches.

\section{MAE \& Precision - Netflix}
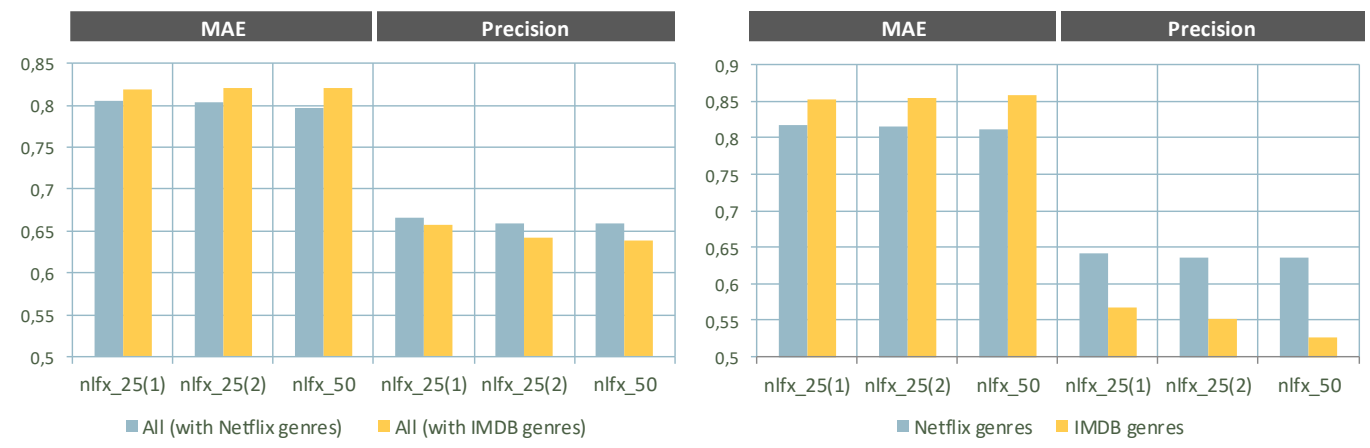

Figure 9 - MAE and Precision comparing algorithm's performance using original Netflix genres against IMDB genres.

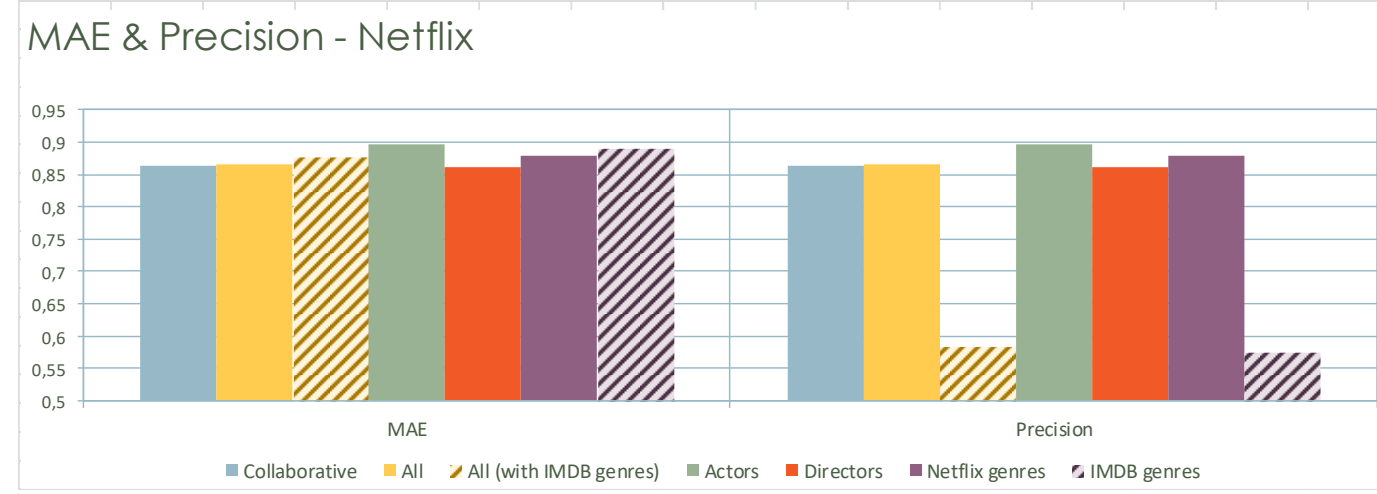

Figure 10 - MAE and Precision comparing algorithm's performance using original Netflix genres against IMDB genres (chronological partition). 


\subsection{Sameness of the top-N results}

Besides the influence of metadata on the quality of the recommendations and how the metric used to compare algorithms can provide different views of the problem, another question arises. Do the approaches that have better performance, recommend the same programs? For example, given that content-based algorithm performs well both when using all the metadata elements and the directors individually, will they recommend the same programs?

Table 8 and Table 9 compare the collaborative and content-based approaches by examining how many of the top-10 and top-20 recommended items are the same for one of the case studies presented in this paper (Netflix dataset, partitioned by the number of ratings per user - subdataset 50\%). The colours in the tables point out the greater similarities achieved for each of the pairs of approaches (e.g. the greater similarity obtained for the collaborative method was obtained with the Directors' based approach 1.91; thus, the intersection of the collaborative line with the director's column is marked in blue). Table 10 and Table 11 present the same analysis for the Movielens dataset.

From the tables, it is clear that different approaches recommend different programs. For the top-20 list, for example, recommendations based on all metadata only share $25 \%$ of the items recommended based on the actors. These results may be relevant in the implementation of hybrid recommenders that list together the results obtained from two or more approaches.

It is also interesting to notice that although the approaches based on all the metadata and on the directors only showed similar performances, they share only 4.02 of the items recommended for the Top20 list of the Movielens dataset (Table 11).

Table 8 Sameness of the top 10 recommendations for Netflix dataset

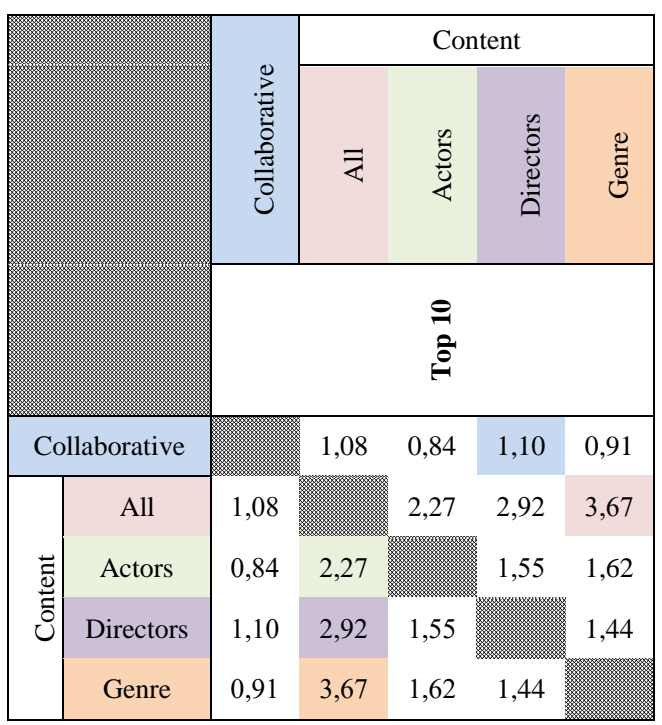

Table 9 Sameness of the top 20 recommendations for Netflix dataset

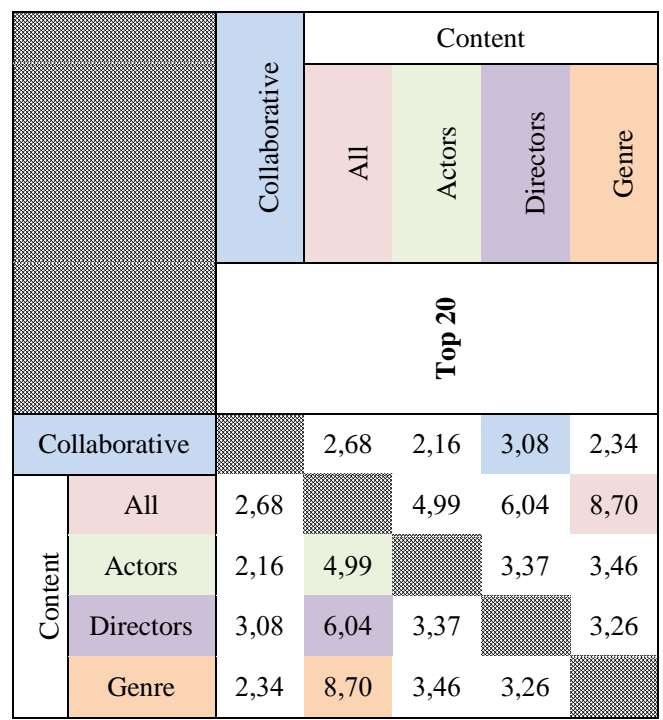


Table 10 Sameness of the top 10

recommendations for Movielens dataset

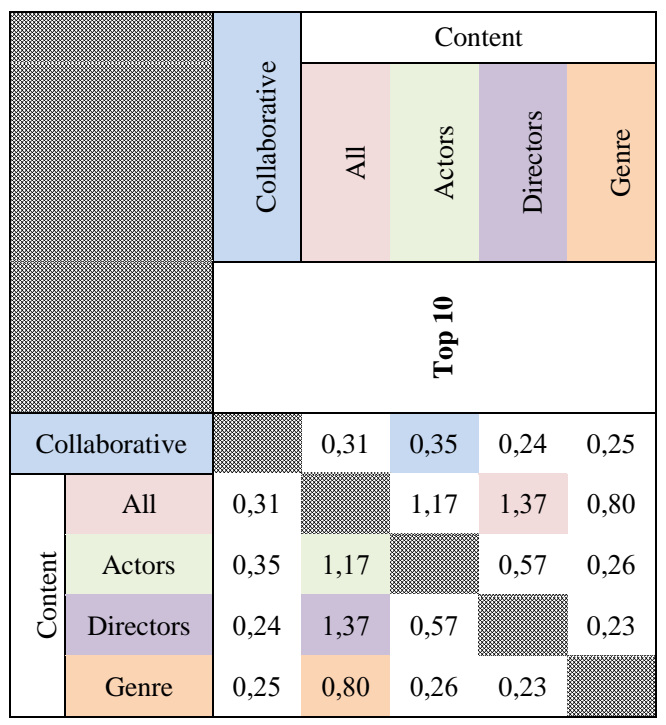

Table 11 Sameness of the top 20

recommendations for Movielens dataset

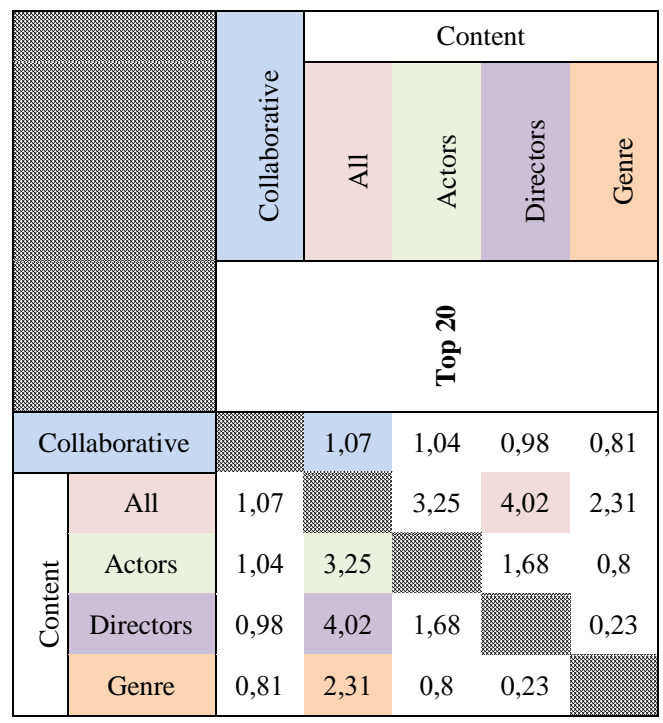

\section{Conclusions}

The work presented in this paper provides a deep evaluation on how content-based recommendation algorithms can be influenced by the metadata information used in the domain of movies and television programs description. Different datasets and metrics were used in order to validate the results and to guarantee that they were not influenced by the dataset used rather than by the metadata itself.

The results presented in this paper demonstrate that although the collaborative algorithm usually performs better, improvements can be achieved in the content-based approach by using the adequate metadata information, making the results quite similar. This may contribute to make the content-based algorithm a good alternative when e.g. computational cost is too high to implement the collaborative method or the information available in the service is sparse and does not enable finding the best neighbours.

The combination of different metadata elements provides usually better results when compared with metadata used separately. In addition, the greater the number of metadata attributes used in combination, the better is the performance. However, an exception occurs in the case of the directors used individually and this finding may help decrease computation time while maintaining the same quality.

The better performance achieved by using information on the directors may be likely explained by the fact that users do not usually guide their interests by generic programs attributes (such as genre) but mainly by a quality perception that is not explicit in the descriptive content. This may be intuitively read from the dataset contents where, for example, films directed by James Cameran always have good ratings (above 7) while 
actors, even with a good reputation, may participate in movies with fairly inconsistent ratings. This leads to the conclusion that a film director can provide specific information on the potential quality of a movie that cannot be described with another set of metadata elements. The importance of this attribute is even clearer when looking at findings resulting from very sparse datasets: although all the other algorithms suffered a significant decrease in performance, using the directors metadata enable still guaranteeing good recommendations. This conclusion is relevant when dealing with recent systems with small history information.

Additionally, the results show that the granularity used (e.g. in the genre) has an impact in the quality of the recommendations. This observation can help media asset managers on choosing a more adequate content description schema.

Furthermore it was noticed that the list of items originated by each of the different approaches, have little in common. This fact illustrates the potential of using hybrid approaches to guarantee the diversity of the recommendations.

Future work includes the analysis of other perspectives on the evaluation of recommendation lists such as the novelty and diversity of the results.

\section{Acknowledgments}

The work presented in this paper was partially supported by Fundação para a Ciência e Tecnologia, through FCT/UTA Est/MAI/0010/2009 and The Media Arts and Technologies project (MAT), NORTE-07-0124-FEDER-000061, financed by the North Portugal Regional Operational Programme (ON.2 - O Novo Norte), under the National Strategic Reference Framework (NSRF), through the European Regional Development Fund (ERDF), and by national funds, through the Portuguese funding agency, Fundação para a Ciência e a Tecnologia (FCT).

\section{References}

1. Adomavicius G, Kwon Y (2007) New Recommendation Techniques for Multicriteria Rating Systems. IEEE Intelligent Systems, 22 (3):pp 48-55.

2. Adomavicius G, Tuzhilin A (2005) Toward the next generation of recommender systems: A survey of the state-of-the-art and possible extensions. IEEE Transactions on Knowledge and Data Engineering 17(3):734-749.

3. Ahn S, Shi CK (2008) Exploring Movie Recommendation System Using Cultural Metadata. In: Proceedings of the 2008 International Conference on Cyberworlds, pp 431-438.

4. Bar-Ilan J,Keenoy K, Yaari E, Levene M (2007) User rankings of search results. Journal of the American Society for Information Science and Technology, 58(9): 1254-1266. 
5. Bar-Ilan J, Mat-Hassan, M., \& Levene, M. (2006). Methods for comparing rankings of search engine results. Computer Networks, 50(10): 1448-1463.

6. Basu C, Hirsh H, Cohen W (1998) Recommendation as classification: Using social and content-based information in recommendation. In: Proceedings of the Fifteenth National Conference on Artificial Intelligence, pp 714-720.

7. Bennett J, Lanning S (2007) The Netflix Prize. In: Proceedings of KDD Cup and Workshop.

8. Bellogin A, Castells P, Cantador I (2011) Precision-oriented evaluation of recommender systems: an algorithmic comparison. In: Proceedings of the fifth ACM conference on Recommender systems, pp 333-336.

9. Bobadill J, Serradilla F, Bernal J (2010) A new collaborative filtering metric that improves the behavior of recommender systems. Knowledge-Based Systems, 23(6):520-528. doi:10.1016/j.knosys.2010.03.009

10. Billsus D, Pazzani MJ (1998) Learning collaborative information filters. In In: Proceedings of the 15th International Conference on Machine Learning, pp 46-54.

11. Breese J S, Heckerman D, Kadie C (1998) Empirical analysis of predictive algorithms for collaborative filtering. In In: Proceedings of the Fourteenth conference on Uncertainty in artificial intelligence, pp 43-52, Morgan Kaufmann, San Francisco.

12. Burke R (2007) Hybrid web recommender systems. In: The Adaptive Web, pp 377-408. Springer, Berlin.

13. Castells P, Vargas S, Wang J (2011) Novelty and Diversity Metrics for Recommender Systems: Choice, Discovery and Relevance. International Workshop on Diversity in Document Retrieval at the 33rd European Conference on Information Retrieval.

14. Chen K, Chen T, Zheng G, Jin O (2012) Collaborative personalized tweet recommendation. In: Proceedings of the 35th international ACM SIGIR conference on Research and development in information retrieval, pp 661-670.

15. Cotter P, Smith B (2000) PTV: Intelligent Personalised TV Guides. In: Proceedings of the 12th Innovative Applications of Artificial Intelligence Conference, pp 957-964.

16. Cremonesi P, Koren Y, Turrin R (2010) Performance of recommender algorithms on top-n recommendation tasks. In: Proceedings of the fourth ACM conference on Recommender systems, pp. 39-46.

17. Cremonesi P, Turrin R, Lentini E, Matteucci M (2008) An evaluation methodology for collaborative recommender systems. In In: Proceedings of the 2008 International Conference on Automated solutions for Cross Media Content and Multi-channel Distribution, pp 224231.

18. Fouss F, Serens M (2008) Evaluating performance of recommender systems: An experimental comparison. In In: Proceedings of the 2008 IEEE/WIC/ACM International Conference on Web Intelligence and Intelligent Agent Technology, 1:pp 735-738.

19. Gorgoglione M, Panniello U, Tuzhilin A (2011) The effect of context-aware recommendations on customer purchasing behavior and trust. In: Proceedings of the fifth ACM conference on Recommender systems, pp 85-92. 
20. Herlocker J, Konstan J, Terveen L, Riedl J (2004) Evaluating collaborative filtering recommender systems. ACM Transactions on Information Systems, 22(1):pp. 5-53.

21. Hsu S H, Wen M, Lin H, Lee C (2007) AIMED-A personalized TV Recommendation System. In In: Proceedings of EuroITV, pp 166-174.

22. Iaquinta L, Gemmis M, Lops P, Semeraro G, Filannino M, Molino P (2008) Introducing Serendipity in a Content-Based Recommender System. In: Proceedings of the 2008 8th International Conference on Hybrid Intelligent Systems, pp 168-173.

23. Jancsary J, Neubarth F, Trost H (2010) Towards context-aware personalization and a broad perspective on the semantics of news articles. In: Proceedings of the fourth ACM conference on Recommender systems , pp 289-292.

24. Jiang M, Cui P, Liu R, Yang Q, Wang F (2012) Social contextual recommendation. In: Proceedings of the 21st ACM international conference on Information and knowledge management, pp 45-54.

25. Koren Y (2010) Collaborative filtering with temporal dynamics. Communications of the ACM, 53(4):89-97. doi:10.1145/1721654.1721677.

26. Koren Y, Bell R, Volinsky C (2009) Matrix factorization techniques for recommender systems. Computer, 42(8):30-37.

27. Koren Y (2008) Factorization meets the neighborhood: a multifaceted collaborative filtering model. In: Proceedings of the 14th ACM SIGKDD international conference on Knowledge discovery and data mining, pp 426-434.

28. Lang K (1995) NewsWeeder: Learning to filter netnews. In: Proceedings of the 12th International Conference on Machine Learning, California, pp 331-339.

29. Lampropoulos AS, Lampropoulos LS, TsihrintzisA GA (2011) Cascade-Hybrid Music Recommender System for mobile services based on musical genre classification and personality diagnosis. In Multimedia Tools and Applications.

30. Lipczak M, Milios E (2010) Learning in efficient tag recommendation. In: Proceedings of the fourth ACM conference on Recommender systems, pp 167-174.

31. Lommatzsch A, Kille B, Albayrak S (2013) A framework for learning and analyzing hybrid recommenders based on heterogeneous semantic data. In: Proceedings of the 10th Conference on Open Research Areas in Information Retrieval, pp 137-140.

32. Miller B N, Albert I, Lam SK, Konstan JA, Riedl J (2003) Movielens Unplugged: Experiences with an Occasionally Connected Recommender System. In: Proceedings of the 8th international conference on Intelligent user interfaces, pp 263-266.

33. Mitchell TM (1997) Machine Learning. McGraw-Hill, Singapore.

34. Moshfeghi Y, Piwowarski B, Jose J (2011) Handling data sparsity in collaborative filtering using emotion and semantic based features. In: Proceedings of the 34th international ACM SIGIR conference on Research and development in Information Retrieval, pp 625-634.

35. Resnick P, Varian H R (1997) Recommender systems. Communications of the ACM 40(3):56-58. 
36. Resnick P, Iacovou N, Suchak M, Bergstrom P, Riedl J (1994) GroupLens: An Open Architecture for Collaborative Filtering of Netnews. In: Proceedings of the 1994 ACM conference on Computer supported cooperative work, pp 175-186.

37. Pazzani M, Billsus D, (1997) Learning and revising user profiles: The identification of interesting web sites. Machine Learning, 27:313-331.

38. Pilászy I, Tikk D (2009) Recommending new movies: even a few ratings are more valuable than metadata. In: Proceedings of the third ACM conference on Recommender systems, pp 93-100.

39. Rendle S, Gantner Z (2011) Fast context-aware recommendations with factorization machines. In: Proceedings of the 34th International ACM SIGIR Conference on Research and Development, pp 635-644.

40. Ricci F, Rokach L, Shapira B, Kantor P, Paul B (2010) Recommender Systems Handbook. Springer, London.

41. Sarwar B, Karypis G, Konstan K, Riedl J (2000) Analysis of recommendation algorithms for e-commerce. In: Proceedings of the 2nd ACM conference on Electronic commerce, pp 158167.

42. Setten MV (2002) Experiments with a Recommendation Technique that Learns Category Interests. ICWI, pp 2712-2718.

43. Soares M, Viana P (2012) TV Recommendation and Personalization Systems: integrating broadcast and video on-demand services. In: Proceedings of the ECUMICT 2012 - European Conference on the Use of Modern Information and Communication Technologies.

44. Symeonidis P, Nanopoulos A, Manolopoulos Y (2007) Feature-weighted user model for recommender systems. User Modeling 2007, Lecture Notes in Computer Science, 4511:97106, Springer.

45. Tang J, Hu X, Gao H, Liu H (2013) Exploiting Local and Global Social Context for Recommendation. In: Proceedings of the Twenty-Third international joint conference on Artificial Intelligence, pp 2712-2718.

46. Vargas S, Castells P (2011) Rank and Relevance in Novelty and Diversity Metrics for Recommender Systems. In In: Proceedings of the fifth ACM conference on Recommender systems, pp 109-116.

47. Wei C, Hsu W, Lee M (2011) A unified framework for recommendations based on quaternary semantic analysis. In: Proceedings of the 35 th international ACM SIGIR conference on Research and development in information retrieval, pp 661-670.

48. Yang B, Lei Y, Liu D, Liu J (2013) Social collaborative filtering by trust. In: Proceedings of the Twenty-Third international joint conference on Artificial Intelligence, pp 2747-2753.

49. Yang X, Steck H, Guo Y, Liu Y (2012) On top-k recommendation using social networks. In: Proceedings of the sixth ACM conference on Recommender systems, pp 67-74.

50. Yu Z, Zhou X, Hao Y, Gu J (2006) TV program recommendation for multiple viewers based on user profile merging. User Modeling and User Adapted Interaction, 16(1):62-82.

51. Zhong E, Fan W, Yang Q (2012) Contextual Collaborative Filtering via Hierarchical Matrix Factorization. SDM, pp 744-755. 
52. Zhou K, Yang S, Zha H (2011) Functional matrix factorizations for cold-start recommendation. In: Proceedings of the 34th international ACMSIGIR conference on Research and development in Information Retrieval, pp 315-324

53. Zhuang Y, Chin WS., Juan, Y.-C., \& Lin, C.-J. (2013). A fast parallel SGD for matrix factorization in shared memory systems. In: Proceedings of the 7th ACM conference on Recommender systems - RecSys 13, pp 249-256. 